\title{
The Design Space of Robots: Investigating Children's Views
}

\author{
Sarah Woods, Kerstin Dautenhahn \\ University of Hertfordshire, School of \\ Computer Science, Adaptive Systems \\ Research Group \\ Hatfield, AL10 9AB, UK \\ s.n.woods, k.dautenhahn@herts.ac.uk
}

\author{
Joerg Schulz \\ University of Hertfordshire \\ Psychology Department \\ Hatfield, AL10 9AB, UK \\ j.schulz@herts.ac.uk
}

\begin{abstract}
Our study considers children's perceptions of robots in terms of physical attributes, personality and emotion traits. To examine children's attitudes towards robots, a questionnaire approach was taken with a large sample of children, followed by a detailed statistical framework to analyse the data. Results show that children clearly distinguish between emotions and behaviour when judging robots. The distinguishing robotic physical characteristics for positive and negative emotions and behaviour are highlighted. Children judge human-like robots as aggressive, but human-machine robots as friendly providing support for the Uncanny Valley. The paper concludes with discussing the results in light of design implications for children's robots.
\end{abstract}

\section{Introduction}

Robots have been used in a variety of service, educational or therapeutic applications, some projects focussing on the robot's functionalities, others on the social aspects, e.g. [1], [2], [3]. Increasingly, research addresses design implications for the desired end usergroup, be it adults, children, experts, or therapeutic client groups. Design specifications encompass a wide range of physical and behavioural features, as well as psychological aspects such as personality components and emotional expressions.

\subsection{Design Considerations for Robots}

Robot Appearance: There is limited research that has directly considered the impact of the design of robot appearance on human-robot interaction cf. categorisations for understanding robot appearance proposed by Fong et al. [4].

Anthropomorphism is the tendency to attribute human characteristics to objects with a view of helping to understand and interpret their actions. It has been argued that for humans to have believable and meaningful interactions with robots then the robot should be structurally and functionally similar to a human, cf. [5].
The tendency to attribute human-like characteristics is viewed as a useful tool to enable engaging human-robot interaction [6], and has led researchers to pursue research into robots that resemble humans as closely as possible. Robot Movement: Little consideration has been given to robot movement and whether this is an underlying design implication related to overall appearance. Related to the concept of robot appearance is the danger of falling into the 'Uncanny Valley' as described by Mashiro Mori [7] who contended that if robots become too close to realism (appearing very human), but are not perfect (indistinguishable from humans), then the imperfections can be viewed extremely negatively.

Robot Gender: Do humans and in particular children assign gender to robots, and does this have an impact on perceptions of robot personality, believability and engagement? Few conclusive results are available [8] [9] [10]. Little is known about the relationship between robot gender and how this impacts on the perception of robot personality although some robotic engineers and designers have remarked that developers and users of gaming software and robots tend to be males which means that female views may have been ignored.

Robot Personality: People use personality in a similar way to other social stereotypes to try to make sense of social behaviour in terms of goals, beliefs and emotions. The tendency for humans to assign personality qualities to robots may facilitate the user to understand its behaviour, and help to shape the user interaction and assist with design restrictions, cf. [11]. Experiments have shown that robot personality should match its design purpose [12]. However, few studies have yet considered whether children's perceptions of robot personality are related to robot appearance.

\subsection{The child as the designer}

Although children are encouraged to take part in a wide range of robotic competitions, robots are still frequently designed from an adult perspective ignoring children's perceptions and attitudes about robots. If successful robots are to be designed and used within 
educational curriculum activities, children should be at the forefront of the design course and suitable methods for obtaining children's views should be designed and utilised, cf. arguments in e.g. [13] [14].

\subsection{A psychological approach into robot attitudes}

The studies by Khan [15] and Scopelliti et al. [16] are among the first to have used a questionnaire approach to explore adults attitudes towards the design of a domestic robot. In this particular study we are examining children's perceptions and attitudes towards different types of robots in terms of robot appearance, physical attributes, and personality and emotional characteristics. A psychological approach is adopted for this study using a questionnaire approach and a quantitative statistical framework to analyse the results. Our research questions are: 1) How do children evaluate different types of robot appearances? 2) Depending on the physical characteristics of the robot, how do children rate the personality and emotional traits of the robots? 3) What are the design implications for robots based on children's perceptions of robots?

\section{Method}

\subsection{Design \& Participants}

This study used a questionnaire based design and quantitative statistical techniques. Children viewed 5 robot images, completing the robotics questionnaire for each image. The independent variables were the physical robot attributes (appearance, movement, colour, shape). The dependent variable(s) were robot personality and emotion traits (friendly, aggressive, angry, shy, bossy, happy, sad). 159 children (male: N:82 (52\%) and girls: N: $77(48 \%)$ aged $9-11$ (years $5 \& 6$ ) participated in the study $(\underline{M}$ age $=$ 10.19 years, SD: 0.55). Four schools based in Hertfordshire, UK, participated in the research with an equal spread of low, middle and upper socioeconomic status. Each child's reading ability was verified before they participated in the study.

\subsection{Instruments}

\section{Robot Pictures}

A number of different internet sources were consulted to compile the robot images portfolio, consisting of 85 standardised images. A coding schedule was used to categorise the robots according to the following criteria: a) movement, b) shape, c) overall appearance (e.g. car, human, machine, animal), d) facial features, e) gender, f) functionality (e.g. toy, friend, machine). Based upon the age and cognitive abilities of the children who took part in the study, 8 groups containing 5 robot images were formed, (total N: 40 robot images). The remaining 35 robot images were excluded from the study due to unclear images etc. We ensured that each robot group included the different robot attributes identified during phase one of the coding scheme (different types of movement, shapes, overall robot appearance, facial features and gender).

Robot Pictures Questionnaire: 'What do you think?'

A questionnaire was designed to enquire about children's perceptions of different robot attributes. Section one referred to questions about robot appearance (e.g. what does this robot use to move around? What shape is the robot's body?). Section two asked questions about robot personality, rated according to a 5-point likert scale and included questions about friendliness, aggressiveness, whether the robot appeared shy, and whether the robot appeared bossy. An example question was: Do you think this robot is (or could be) aggressive?

\subsection{Procedure}

Groups of between 4-8 children were seated in such a way that they would be able to answer the questionnaires confidentially without distraction from other children. A set of 5 robot images ( 1 of 8 categories $A-E$ ) were distributed to each child. Each child completed 5 copies of the Robot Pictures Questionnaire for each of the images.

\subsection{Statistical Analysis}

Each robot image (N: 40) was rated between 18-22 times. Pearson correlations were first carried out to examine the levels of consistency between child responses for robot personality and emotional traits, and to provide an indication of child comprehension of the robot questionnaire. Chi-square analyses were then carried out to explore children's views of robot feelings and understandability. Next, Principal Components Analysis was carried out for robot personality and emotional traits to determine whether subsequent analyses could be carried out on a reduced number of meaningful factors. Based on the two factors which emerged, a ranked line graph based on standardised Z-scores was produced for each robot ID to explore for potential physical distinguishing features of the different robots. Cut-off points were then defined and the physical descriptors for the robots that fell within the cut-off points in relation to personality and emotional traits were interpreted. Finally, the percentage level of agreement between children's responses for overall robot appearance (i.e. human, machine, animal) was examined for particular patterns and cognitive mapping.

\section{Results}

Child comprehension of the robot questionnaire: The results from Pearson correlations revealed that all the correlations fell in the right direction indicating that children had good levels of comprehension completing the robots picture questionnaire.

Do robots have feelings? Highly significant differences were found according to children's evaluations of whether a robot had feelings or not $(x=96.72, \mathrm{df}=39(795), \mathrm{p}=$ 
0.000 ) indicating that children's opinions of whether a particular robot had feelings or not were consistent. Out of the 5 robots which each child had to rate, over two thirds of the robots were rated by the children as having feelings. Children were extremely clear in their distinction between a machine looking robot not having any feelings and human looking robots as possessing feelings. Animal looking robots did not feature among the extreme standardised residual scores. E.g. robot number 31 was rated by children as having an overall human-like appearance and this was rated by $86 \%$ of children as having feelings. In contrast, robot number 61 which was viewed as a machine was rated by $67 \%$ of children as having no feelings.

Do robots understand children? Children were asked whether they believed particular robots would understand them if they tried to talk to it. Cross-tabulations revealed a significant result $(x=169.75, \mathrm{df}=39,(795), \mathrm{p}=0.000)$ indicating that children's views of whether a particular robot would understand them were consistent. Children had clear opinions as to whether a robot would understand them or not making a distinction between machine looking robots not being able to understand them and human and animal looking robots being able to understand them. E.g. robot number 79 which was rated by children as having a human-like appearance was rated by $90 \%$ of children as being able to understand them. Conversely, robot number 94 which was classified by children as having a machine like appearance was rated by $90 \%$ of children as not being able to understand them.

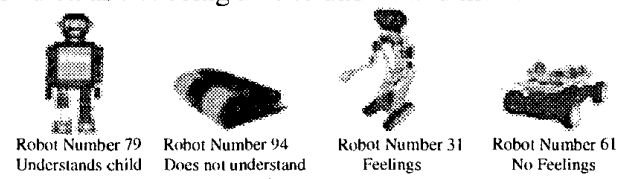

Robot personality dimensions: Principal Components Analysis (PCA) was carried out including child ratings according to the 5-point likert scale for friendliness, aggressiveness, shyness, bossiness, happiness, sadness, anger and fright. The best solution from the PCA was Varimax Rotation for gender combined. Two factors emerged with eigenvalues of 4.99 and 1.63 accounting for $82.84 \%$ of the total variance. Factor I accounted for $62.41 \%$ of variance and Factor II $20.43 \%$ of variance. All variables loaded onto the primary factors at .80 or greater. Factor I had 6 primary loadings and constituted robotic behaviours with intent and was termed 'Behavioural Intention' (BI). These included friendliness, aggressiveness, shyness, bossiness, anger and fright. Factor II had 2 primary factors and was termed 'Emotion' (E) as it referred to the bi-polar emotions of happiness and sadness. Two dimensions were then computed for 'Behavioural Intention' and 'Emotion' including component scores with loadings of .50 or more. Z-Scores were computed for the two factor-based scales to allow for standardised comparisons between each of the factors.

Robot appearance and personality traits: To explore potential distinguishing physical robotic characteristics in relation to BI and E, a scatterplot (Figure 1) was produced. A cut-off point (indicated by dashed line) of $+/-0.5$ Standard Deviations was used for BI and a slightly less stringent cut-off point of $+/-0.4$ was used for $E$ to examine the most extreme robots.

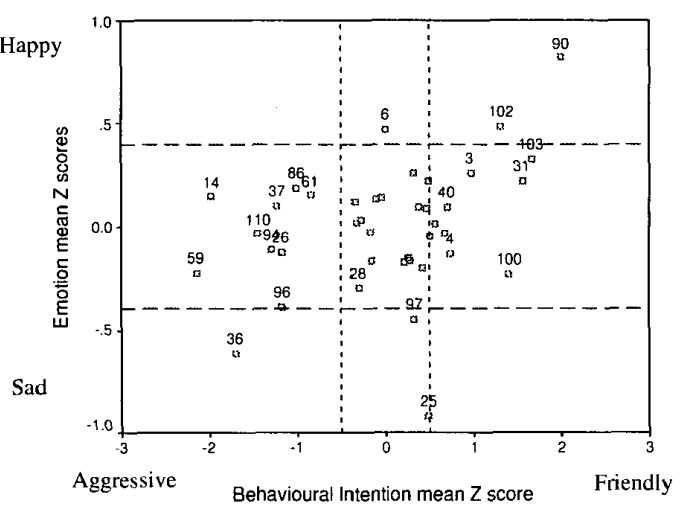

Figure 1: Scatterplot illustrating Mean Z-scores for Emotion and Behavioural Intention for each robot id (N:40).

Figure 1 illustrates those robots that fell beyond the defined cut-off points. The majority of robot images rated by children had similar mean Z-scores for $E$ resulting in indistinguishable physical robot characteristics. However, robots 6,102 and 90 were rated by the children as being the happiest, whilst robots $25,36,97$, and 96 , were rated as the saddest robots, (see table 2 for robot images). In contrast, for BI there were more robots that fell within the defined cut-off points, (see table 3 for images). Table 4 illustrates the robots that fell within the cut-off points $(+/-$ $0.4 / 0.5$ ) for E and BI and distinguishable physical characteristics. Robotic physical characteristics associated with sadness were having 2 legs, a rectangular/square body shape, having a human like appearance, facial features and male gender. Physical robotic attributes for happiness were harder to distinguish. However, children's ratings of happiness were associated with an animal or human like appearance, facial factures, and male or female gender as opposed to having no gender. Robots rated as having negative BI were associated with having legs or wheels, having a rectangular body shape, having a machine like appearance and a male gender. Facial features were indistinguishable for negative BI. Robots rated as having positive BI were distinguished as having legs, facial features and a male or female gender. Robot appearance was indistinguishable for positive BI. Robot images that fell within the specified cut-off points revealed that those robots with positive BI had a cartoon-like appearance, exaggerated facial features in particular the 
eyes, used legs or wheels as the form of movement and were brightly coloured.

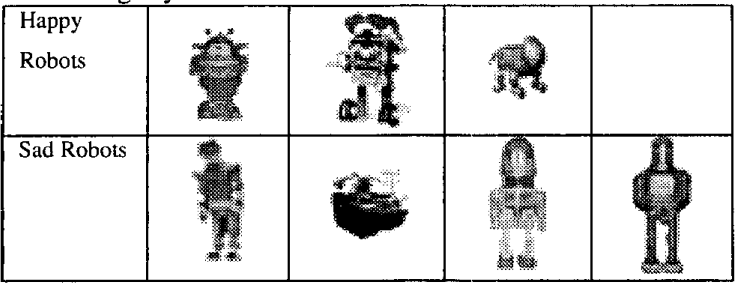

Table 2: The happiest and saddest robots as rated by children.

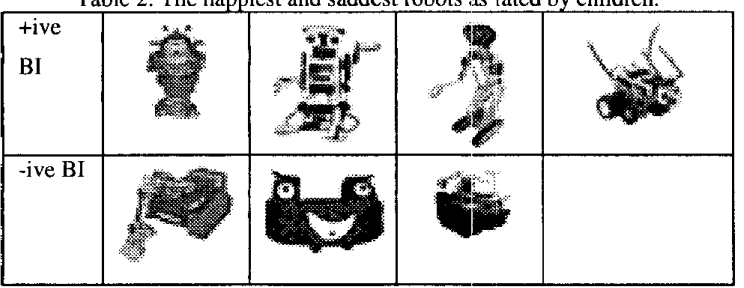

Table 3: Robots with highest levels of positive and negative behavioural intention according to child rating

\begin{tabular}{|c|c|c|c|c|}
\hline & $\begin{array}{l}\text { Emotion } \\
\text { Sadness } \\
\end{array}$ & $\begin{array}{c}\text { Emotion } \\
\text { Happy }\end{array}$ & -ive B.I & tive B.I \\
\hline \multicolumn{5}{|c|}{ Movement } \\
\hline 2 legs & $\mathbf{m}$ & \multirow{4}{*}{ Indistinguishable } & $\mathbf{w}$ & $\mathbf{\square}$ \\
\hline Wheels & & & 五 & \\
\hline Tracks & & & & \\
\hline 4 legs & & & & E \\
\hline \multicolumn{5}{|c|}{ Body shape } \\
\hline Circ/oval & & \multirow[t]{2}{*}{ Indistinguishable } & & \\
\hline Rect/sq & w & & - & - \\
\hline \multicolumn{5}{|c|}{ Looks like } \\
\hline \multicolumn{5}{|l|}{ Animal } \\
\hline \multicolumn{5}{|l|}{ Human } \\
\hline Machine & & & E & प् \\
\hline \multicolumn{5}{|c|}{ Facial Features } \\
\hline Face & $=$ & ש & U & E \\
\hline No face & & & $\mathbf{E}$ & \\
\hline \multicolumn{5}{|c|}{ Gender } \\
\hline Male & E5 & $\mathbf{\theta}$ & 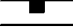 & E \\
\hline Female & & E & & - \\
\hline No gender & & & & \\
\hline
\end{tabular}

Table 4: Summary of the physical robotic features distinguishing positive and negative Behavioural Intention and Emotion.

In contrast, robots with negative $B I$ had a realistic-like appearance, used legs or wheels, had less exaggerated facial features or faces which were partially camouflaged, and had dull colours such as grey, brown and black. Robots rated as being happy (+ emotion) had a cartoonlike appearance, had legs, and had exaggerated facial features. Conversely, robots rated as being sad (- emotion) had a realistic-like appearance and some had enclosed heads (i.e. head concealed behind a capsule or helmet).

\begin{tabular}{|l|c|c|}
\hline & Cartoon Appearance & Realistic Appearance \\
\hline Behavioural Intention & Positive & Negative \\
\hline Emotion & Positive & Negative \\
\hline
\end{tabular}

Table 5: Robot appearance and the association with positive or negative behavioural intention and emotions

Overall robot appearance: Three new variables were calculated based on the percentage agreement between children's responses for robot appearance (animal, machine, human). Pearson correlations revealed significant negative correlations between the 3 robot appearances. A 3-D scatterplot (figure 2) was then produced to explore the systematic association between robot appearance by plotting the variables human-like, machine-like and animal like.

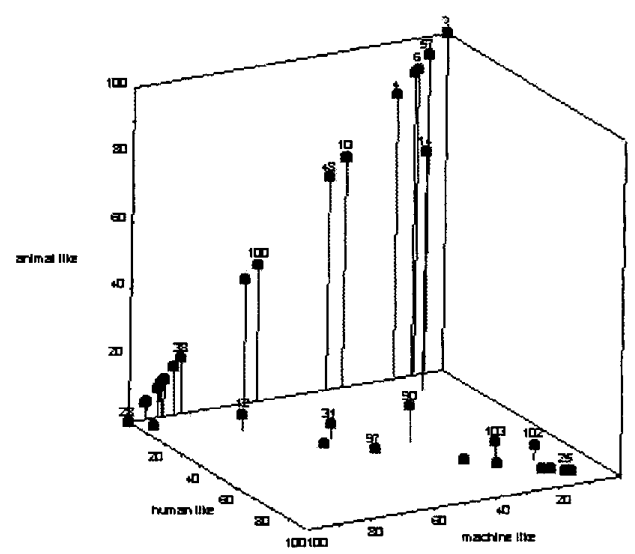

Figure 2: Robots classified by children as pure animals, machines or a mixture of human-machine/animal-machine (for presentation purposes not all 40 robot id numbers are shown)

Figure 2 illustrates for example that children rated robot id number 3 as having $100 \%$ animal-like features, robot id 28 had $100 \%$ pure machine-like features, robot id number 102 had $80 \%$ human-like and $20 \%$ machine-like and robot id 97 had 50\% human-like features and 50\% machine-like features (table 6 for images).

A one-way ANOVA between robot appearance and emotion revealed a non-significant trend $(\mathrm{F}=1.97, \mathrm{df}=4$ (N40), $\mathrm{p}=0.12$ ). Post-hoc comparisons demonstrated that the biggest mean difference lay between pure animal-like and pure machine-like robots $(\underline{\mathrm{M}}$ animal $=0.81, \underline{\mathrm{M}}$ machine $=0.36, p=0.21$ ) indicating that pure animal-like robots were rated by children as being happier than pure machine-like robots. A small significant finding was revealed between robot appearance ratings and children's perceptions of robot $\mathrm{BI}(\mathrm{F}=2.51, \mathrm{df}=4(\mathrm{~N} 40), \mathrm{p}=0.06)$. Post-hoc comparisons revealed the largest mean differences between human-like robots and humanmachine robots $(\underline{M}$ pure human $=-0.39, \underline{M}$ humanmachine $=0.71$ ) and machine-like robots and humanmachine robots ( $\mathrm{M}$ pure machine robots $=-0.31$ ). Humanlike robots had the highest mean negative BI scores indicating that children perceived these robots as the most aggressive and bossy, and a combination of animalmachine or human-machine like as the most friendly and shy. Figure 3 illustrates children's perception of robot appearance and their judgements of a robot's BI in relation to the Uncanny Valley proposed by Mori [7]. According to our interpretation, children's judgements provide support for the Uncanny Valley that as a robot increases in humanness there is point when the robot is not $100 \%$ 
similar to humans and the balance between humanness and robotness becomes uncomfortable. For example robots rated by the children as being human-machine like in appearance (no. 4) had the highest positive scores for BI and fall mid-way between familiarity and similarity. In contrast, robots rated by the children as human-like in appearance were rated as having the most negative BI scores which indicate that they fall into the Uncanny Valley and children found these types of robots more uncomfortable.

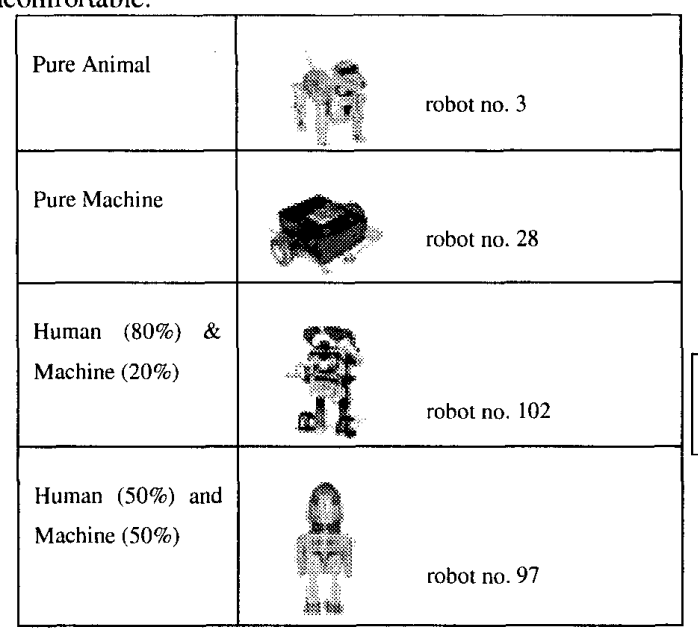

Table 6: Robot appearance for pure animals, pure machines and combinations of human/machine

\section{Discussion}

The major aims of this exploratory study were to examine how children evaluated different types of robots and whether specific robot physical attributes were related to distinct personality and emotional traits. A summary of the main results indicated: a) Children had consistent opinions towards different robots in terms of whether they had feelings or whether they thought robots could understand them. b) Two dimensions emerged from children's evaluations of robot behaviour. These were termed 'Emotion' and 'Behavioural Intention'. c) Emotion constituted the bi-polar emotions happiness and sadness and Behaviour Intention was made up of friendliness, aggressiveness, shyness, bossiness, anger and fright. d) Sad robots were characterised as having 2 legs, a rectangular body, human-like appearance, facial features and male gender. e) Happy robots as judged by children had animal-like or human-like appearance, facial features and a male or female gender. f) Aggressive, bossy and angry robots were characterised by children as using 2 legs or wheels as the form of movement, rectangular body, machine-like appearance, and male gender. g) Friendly, shy and fearful robots were classified as using legs for movement, having a rectangular body, facial features and a gender. h) Overall robot appearance was an important characteristic for children with pure animal robots rated as the happiest and pure machine robots rated as the most aggressive and angry. i) Animal-machine and humanmachine robots were rated by children as being the most friendly.

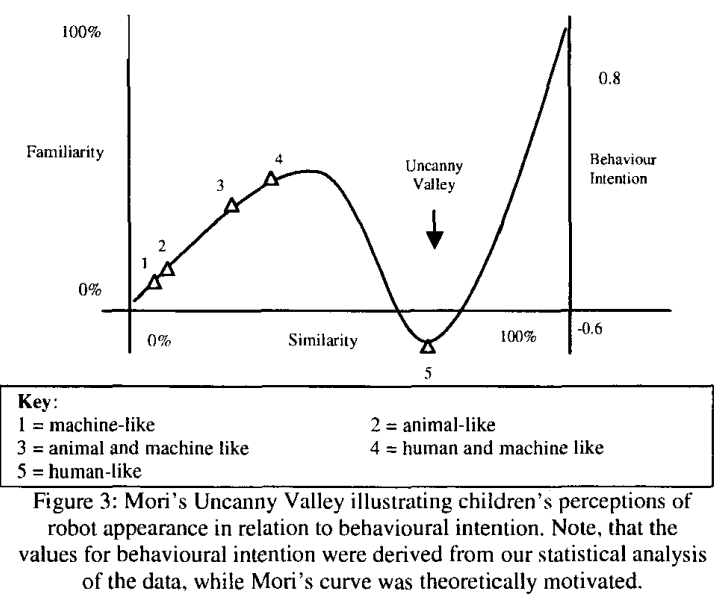

\subsection{Design implications for children's robots}

The results of this study are in line with those proposed earlier [13] [14] that valuable input can be gained from including children in the design phase of a research project as their ideas can be very different compared to an adult perspective. This is particularly true for primary school aged children who have less developed cognitive and social skills compared to adults and are likely to judge things along simpler cognitive and social dimensions.

An important observation from the findings was that facial features, male gender, and robot body shape did not enable distinctions to be made between friendly, aggressive, sad and happy robots as robots in all of these categories possessed facial features, a male gender and a rectangular body. These results indicate that it is important for robot designers to consider a combination of physical characteristics rather than focusing specifically on certain features in isolation. For example, many designers focus predominantly on the different types of facial features and expressions needed for a robot whilst ignoring robot movement and body shape. In contrast to [8], the current results highlighted that children aged 9-11 years assigned a gender to the robot images, in particular male gender. It was interesting that female gender was associated with positive robot traits such as happiness and friendliness, whereas male gender was not distinguishable for positive and negative robot traits. 
Different from the widespread assumption that robots interacting with humans should resemble humanness as closely as possible, the present results indicated that children felt some discomfort towards the images rated as human-like. This was illustrated in figure 3 where human-like robots fell into the uncanny valley for negative behavioural intention. In contrast, human-machine like robots were rated the most positively, providing support for the theory that as a robot increases in humanness, there is a point when the robot is not $100 \%$ similar to human and the balance becomes uncomfortable. The design implications can be summarised as follows: 1) Robots should have cartoon like features, exaggerated facial features, a female gender and be brightly coloured for positive behaviours. 2) Robots should have realistic features, less clear facial features, and be dully coloured to depict negative behaviours. 3) The whole appearance of a robot should be considered at the outset of the design phase rather than focusing on specific aspects such as the face. 4) Robots for children should not be designed to look completely human-like. A mixture of human-machine features is most desirable.

\section{Conclusion}

This exploratory study has provided a clearer understanding about children's views of robots in terms of positive and negative behaviour and how these behaviours relate to a robot's appearance.

Several strengths and weaknesses of the present study need to be addressed in future work. Some of the children had seen some of the images before -- e.g. in robot wars this may have influenced their perceptions if they had familiarity with them. Questionnaires have be shown to be economical and can be used with a large sample size of children. However, behavioural data of children encountering physical robots are needed to confirm our findings [17]. Reliance on robot photographs makes it difficult to set the context and relate appearance to actual robot behaviour and interaction. However, this avoids behavioural stereotypes and allowed us to just consider appearance separately from behaviour. The next step in our work will be to have some robot demos or videos to determine whether we get the same findings when children are shown real robots that are actually moving. Our work demonstrates the advantages of a psychological perspective towards designing robots for children or other target groups.

\section{References}

[1] S. Thrun, et al., "Probabilistic algorithms and the interactive museum tour-guide robot Minerva," Journal of Robotics Research, vol. 19, pp. 972-1000, 2000.

[2] E. Falcone, R. Gockley, E. Porter, and I. Nourbakhsh, "The personal rover project: The comprehensive design of a domestic personal robot," Robotics and Autonomous Systems, vol. 42, pp. 245-258, 2003.

[3] K. Severinson-Eklundh, A. Green, and H. Hüttenrauch, "Social and collaborative aspects of interaction with a service robot," Robotics and Autonomous Systems, vol. 42, pp. 223-234, 2003.

[4] T. Fong, I. Nourbakhsh, and K. Dautenhahn, "A survey of socially interactive robots," Robotics and Autonomous Systems, vol. 42, pp. 143-166, 2003.

[5] C. L. Breazeal, Designing sociable robots. Massachusetts: The MIT Press, 2002.

[6] B. Friedman, P. H. Kahn (Jr.), and J. Hagman, "Hardware companions? What online AIBO discussion forums reveal about the human-robotic relationship," Digital Sociability, vol. 5, pp. 273-280, 2003.

[7] K. Dautenhahn, "Design spaces and niche spaces of believable social robots," presented at Int. Workshop on Robots and Human Interactive Communication, 2002.

[8] K. E. Bumby and K. Dautenhahn, "Investigating children's attitudes towards robots: A case study," presented at Cognitive Technology Conference, San Francisco, USA, 1999.

[9] B. Reeves and C. Nass, The media equation. Cambridge, UK: Cambridge University Press, 1996.

[10] C. Nass and Y. Moon, "Machines and mindlessness: Social responses to computers," Journal of Social Issues, vol. 56, pp. 81-103, 2000.

[11] D. A. Norman, "How might people interact with agents," Communications of the ACM, vol. 37, pp. 68$71,1994$.

[12] J. Goetz and S. Kiesler, "Cooperation with a robotic assistant," presented at $\mathrm{CHI} 02$ Conference on Human Factors in Computing Systems, New York, USA, 2002.

[13] A. Druin, "Cooperative Inquiry: Developing new technologies for children with children," presented at CHI'99, Pittsburgh, USA, 1999.

[14] M. Scaife and Y. Rogers, "Informing the design of a virtual environment to support learning in children," International Journal of Human-Computer Studies, vol. 55 , pp. 115-143, 2001.

[15] Z. Khan, "Attitude towards intelligent service robots," NADA KTH, Stockholm 1998.

[16] M. Scopelliti, M. V. Giuliani, A. M. D'Amico, and F. Fornara, "If I had a robot at home.... Peoples' representation of domestic robots," in Designing a more inclusive world, S. Keates et al., Eds. Cambridge, UK: Springer, 2004, pp. 257-266.

[17] P. H. Kahn (Jr.), B. Friedman, D. R. Perez-Granados, and N. G. Freier, "Robotic pets in the lives of preschool children," presented at CHI, 2004. 